\title{
Academic Facilitators' Opinions of an Integrated Interprofessional Therapeutics and Prescribing Session for Undergraduate Pharmacy and Medicine Students
}

Authors: Dai John, ${ }^{1}$ Sion Coulman, ${ }^{1}$ Louise Hughes, ${ }^{1}$ Efi Mantzourani, ${ }^{1}$ Rhian Deslandes, ${ }^{1}$ Pamela Bradley, ${ }^{1}$ Leanne Roberts, ${ }^{1}$ Simon Wilkins, ${ }^{1,2,3}$ JamieHayes, ${ }^{3}$ John Thompson, ${ }^{2}$ James Coulson, ${ }^{2}$ Helen Sweetland, ${ }^{2}$ Philip Routledge ${ }^{2}$

${ }^{1}$ School of Pharmacy and Pharmaceutical Sciences, Cardiff University, ${ }^{2}$ School of Medicine, Cardiff University and ${ }^{3}$ Welsh Medicines Resource Centre, Cardiff.

Background: A new interprofessional education (IPE) case-based session which integrated therapeutics, prescribing and communication was developed and piloted. Sessions involved year 3 medicine students working together with year 3 or year 4 pharmacy students. Students played the roles of prescriber and patient in medicines history-taking, adverse drug reaction reporting and prescription writing. Over 700 students and 12 faculty have participated in the academic years 2011/12 and $2012 / 13$. Six cases have been used. The aim was to capture the views of faculty involved with facilitation of these sessions.

Method: Facilitators provided views using a feedback form which requested comments about the cases and the sessions, that is, what went well, suggestions for improvements and any other comments.

Results: Feedback was provided by all 12 facilitators. Positive aspects included the content and problems within the cases, the relevance to both pharmacy and medicine students, the enthusiasm of students and that the students recognised and appreciated each other's strengths and limitations. Faculty were pleased students reported the session as interesting, useful and enjoyable. Areas for improvement include allocation of students to pairs, the venue and scheduling of sessions. Overall, the academics enjoyed facilitating and all wished to continue their involvement. Another benefit is the closer collaboration of some individuals from the different professions with regard to undergraduate learning, teaching and assessment. Furthermore, this new IPE initiative was highlighted as an example of good practice by the General Medical Council in their 2012 post-visit report.

Discussion: In conclusion, academics strongly endorsed the value of these IPE therapeutics and prescribing sessions and support further development of IPE between undergraduate pharmacy and medicine. The collaborative development and delivery of the IPE sessions has strengthened established links and created new relationships between colleagues from both professions. Additional IPE is currently being planned to include other professions. 